\title{
Exploiting Low-rank Structure for Discriminative Sub-categorization
}

\author{
Zheng $\mathrm{Xu}^{1}$ \\ xuzhustc@gmail.com \\ Xue Li ${ }^{1}$ \\ echolixue@gmail.com \\ Kuiyuan Yang ${ }^{2}$ \\ kuyang@microsoft.com \\ Tom Goldstein ${ }^{1}$ \\ tomg@cs.umd.edu
}

\author{
${ }^{1}$ Department of Computer Science, \\ University of Maryland, \\ College Park, USA \\ ${ }^{2}$ Microsoft Research, \\ Beijing, China
}

In visual recognition, sub-categorization, which divides a category into some sub-categories, has been proposed to deal with large intra-class variance in the real world. Recent discriminant sub-categorization approaches utilize samples that do not belong to the category under consideration as negative data for supervision, and cluster positive samples of the category into sub-categories, then simultaneously train the corresponding classifier for each sub-category $[2,4]$. In the jointly clustering and classification framework of previous methods, the classifier for each sub-category is trained by using samples hard-assigned to the sub-category. However, some samples would contribute to the training of several sub-categories since the intra-variance of a category is caused by complex factors. Moreover, sub-categories are closely related since they are discovered from the same category, and the common information among these sub-categories is beneficial for classifier training.

We propose a new approach for discriminative sub-categorization, which adopts the exemplar based method to address the intra-variance in category, and exploits the low rank structure to preserve common information while discovering sub-categories. Our approach builds up the exemplar-LDAs [3], which generates a set of exemplar classifiers with each classifier trained by a single positive sample and all the negative samples. The extreme case of sub-category is to have only one positive sample, which is a compact set for training and modeling. We adopt exemplar classifiers to represent the compact sub-categories and preserve intra-variance in a category. In order to share common information among exemplar classifiers while preserving diversity, we jointly train the exemplar-LDAs for all the positive samples and introduce the trace-norm regularizer on the matrix of weights, as we assume the weights lie on a union of subspaces such that the matrix of weights is low-rank.

We formulate the proposed low-rank least squares exemplar-LDAs (LRLSE-LDAs) as follows. Let $\mathbf{X}_{1}=\left[\mathbf{x}_{1}^{+}, \ldots, \mathbf{x}_{n}^{+}\right]$and $\mathbf{X}_{2}=\left[\mathbf{x}_{1}^{-}, \ldots, \mathbf{x}_{m}^{-}\right]$ denote the centered data matrix ${ }^{1}$ for positive samples and negative samples, $\mathbf{W}=\left[\mathbf{w}_{1}, \ldots, \mathbf{w}_{n}\right]$ denote the weight matrix where each $w_{i}$ is the weight vector of exemplar-LDA for a positive sample. The objective function for training the exemplar-LDAs of positive samples together is

$$
J_{L S E-L D A s}(\mathbf{W})=\frac{\delta}{2}\|\mathbf{W}\|_{F}^{2}+\frac{1}{2}\left\|\mathbf{X}_{2}^{\prime} \mathbf{W}\right\|_{F}^{2}-\operatorname{trace}\left(\mathbf{X}_{1}^{\prime} \mathbf{W}\right)
$$

where $\|\cdot\|_{F}$ is the Frobenius norm of a matrix, $\operatorname{trace}()$ represents the trace of a matrix. We minimize the least squares form in Eq. 1 instead of maximizing the Fisher criterion so that the objective function is convex, inspired by [6]. Eq. 1 has closed-form solution as

$$
\mathbf{W}=\left(\mathbf{X}_{2} \mathbf{X}_{2}^{\prime}+\delta \mathbf{I}\right)^{-1} \mathbf{X}_{1}
$$

where $\mathbf{I}$ is the identity matrix. To discover the structure of sub-categories, we jointly learn the weight for positive samples/exemplars of the category and regularize the weight matrix with a low-rank constraint. Finally, we arrive at the objective function of LRLSE-LDAs,

$$
J_{L R L S E-L D A s}(\mathbf{W})=\xi\|\mathbf{W}\|_{*}+J_{L S E-L D A s}(\mathbf{W})
$$

$\|\cdot\|_{*}$ is the trace norm used to regularize the weight matrix, which is a convex approximation of the rank of a matrix

To solve the convex formulation in Eq. 3, we propose an efficient algorithm based on the scaled form of alternating direction method of multipliers (scaled ADMM) [1]. We reformulate minimizing $J_{\text {LRLSE-LDAs }}(\mathbf{W})$

${ }^{1}$ Data matrix is centered by subtracting the mean of training samples from each sample. We use mean of negative samples to approximate the mean of all negative sample and a positive sample for each exemplar classifier. in Eq. 3 as an equality-constrained convex optimization problem by introducing an intermediate variable $\mathbf{F}$,

$$
\min _{\mathbf{W}, \mathbf{F}} J_{L S E-L D A s}(\mathbf{W})+\xi\|\mathbf{F}\|_{*} \quad \text { s.t. } \mathbf{W}=\mathbf{F}
$$

The augmented Lagrangian for the formulation in Eq. 4 can be written as:

$$
L(\mathbf{W}, \mathbf{F}, \boldsymbol{\Lambda})=J_{L S E-L D A s}(\mathbf{W})+\xi\|\mathbf{F}\|_{*}+\frac{\tau}{2}\left(\|\mathbf{W}-\mathbf{F}+\boldsymbol{\Lambda}\|_{F}^{2}-\|\boldsymbol{\Lambda}\|_{F}^{2}\right)
$$

where $\Lambda$ is the scaled dual parameter matrix, and $\tau$ is the penalty parameter. We iteratively update variables $\mathbf{W}, \mathbf{F}, \boldsymbol{\Lambda}$ as in scaled ADMM, where $\mathbf{W}, \mathbf{F}$ are updated by solving two subproblems both with closed-form solutions, and $\boldsymbol{\Lambda}$ is updated by dual ascent. The two subproblems are

$$
\begin{aligned}
& \mathbf{W}=\arg \min _{\mathbf{W}} J_{L S E-L D A s}(\mathbf{W})+\frac{\tau}{2}\|\mathbf{W}-\mathbf{F}+\boldsymbol{\Lambda}\|_{F}^{2} \\
& \mathbf{F}=\arg \min _{\mathbf{F}} \xi\|\mathbf{F}\|_{*}+\frac{\tau}{2}\|\mathbf{W}-\mathbf{F}+\boldsymbol{\Lambda}\|_{F}^{2}
\end{aligned}
$$

where Eq. 6 has a closed-form solution benefits from the least squares form and Eq. 7 can be solved by singular value thresholding method.

After training the weights of LRLSE-LDAs, we utilize those exemplar classifiers to perform sub-category discovery and visual recognition. For sub-category discovery, we adopt spectral clustering with affinity matrix defined by the prediction scores on positive samples. For visual recognition, we adopt the cross domain recognition approach in [5] by fusing the top-K prediction scores from trained exemplar classifiers.

We conduct comprehensive experiments on various datasets to validate the effectiveness and efficiency of our approach in sub-category discovery and visual recognition. We follow the experimental setting in [4] to evaluate the performance of sub-category discovery. We conduct experiments on ten public datasets from the UCI repository and MNIST, which cover a large variant types of data. LRLSE-LDAs based clustering achieves promising results measured by purity on those datasets. We follow the experimental setting in [5] to evaluate the performance of visual recognition. We use the Office-Caltech dataset for object recognition and the IXMAS dataset for action recognition. LRLSE-LDAs based classification achieves order-of-magnitude speedup with matching performance comparing with state-of-the art in [5].

[1] Stephen Boyd, Neal Parikh, Eric Chu, Borja Peleato, and Jonathan Eckstein. Distributed optimization and statistical learning via the al-

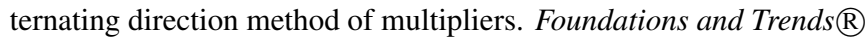
in Machine Learning, 3(1):1-122, 2011.

[2] Pedro F Felzenszwalb, Ross B Girshick, David McAllester, and Deva Ramanan. Object detection with discriminatively trained part-based models. Pattern Analysis and Machine Intelligence, IEEE Transactions on, 32(9):1627-1645, 2010.

[3] Bharath Hariharan, Jitendra Malik, and Deva Ramanan. Discriminative decorrelation for clustering and classification. In ECCV. 2012.

[4] Minh Hoai and Andrew Zisserman. Discriminative subcategorization. In CVPR, 2013.

[5] Zheng Xu, Wen Li, Li Niu, and Dong Xu. Exploiting low-rank structure from latent domains for domain generalization. In ECCV, 2014.

[6] Jieping Ye. Least squares linear discriminant analysis. In $I C M L$, 2007. 